\title{
Using Motor Imagery Training to Improve Reach Estimation in the Elderly: Implications for Reducing Fall Risk
}

\author{
Daniella Mendez Azuela ${ }^{1}$, Ashley Fox ${ }^{2}$, Alberto Cordova ${ }^{1}$ and Carl Gabbard ${ }^{2 *}$ \\ ${ }^{1}$ University of Texas - San Antonio, Department of Kinesiology, Health \& Nutrition, USA \\ ${ }^{2}$ Texas A\&M University, Department of Health \& Kinesiology, USA
}

*Corresponding author: Carl Gabbard, Professor, TAMU 4243 College Station

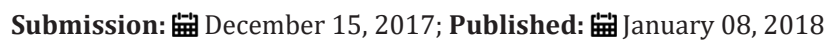

\begin{abstract}
According to recent Center for Disease Control and Prevention statistics, falls are one of the main causes of accidents and fatalities among the elderly. Whereas a several factors may contribute to falls, it has been suggested that weak mental representation of intended actions is a factor. For example, in a reach setting, many older adult's over- or underestimation reach abilities, thus posing a higher risk due to loss of postural control. The intent of this study was to determine if a reach-specific motor imagery training program could improve reach planning and potentially reduce fall risk. The present study involved a group of 23 older adult participants, aged 65-81 years, divided into three groups: a control group and two intervention groups categorized by age, 65- to 73 years and 74- to 81 years. Intervention groups were administered a reach-specific imagery training program three days a week over the course of 4 weeks. Participants were pre- and post-tested on estimation of reach via use of motor imagery in three conditions: seated, standing-on-2-feet, and standing-on-1-foot. Results indicated that both intervention groups significantly improved their reach estimation, $\mathrm{p}<.05$, whereas the control group scores did not differ. No noticeable difference was seen between the two intervention groups or between reach conditions. These findings suggest that motor imagery training has promise as ineffective tool in reducing fall risk among the elderly.
\end{abstract}

Keywords: Motor imagery training; Elderly, Falls

\section{Introduction}

According to the Center for Disease Control and Prevention, "one out of three older adults (those aged 65 or older) fall each year [1]". Falls contribute to the highest number of unintentional injuries experienced by the elderly aged 65 years and older. Furthermore, in the next 17 seconds, an older adult will be treated for fall-related injuries, and in the next 30 minutes, an older adult will die from fall-related injuries [2]. The risk of falling continues to increase the older one gets, and over half of elderly adults aged 80 years fall yearly [1]. Even though these statistics are disturbing, the actual rate of falling occurrences is even greater, due to many incidents not being reported. Clearly, falling has significant implications for quality of life in our aging population.

Research findings tell us that the elderly lack efficient action planning (motor planning) and that the most common reason for falls among the elderly is incorrect transfer or shift of bodyweight, like leaning too far from one's base for support [3]. From these reports, it can be concluded that reaching, especially inefficient reach planning such as over- or underestimation, can cause one to lean too far from their base of support, therefore increasing the risk of falling. In other words, many falls in the elderly can be directly linked to reaching.
Motor planning is used to estimate whether an object is reachable or not from one's current position. Research findings tell us that as one ages, one's mental representation for action planning become less accurate and effective. For example, Gabbard \& Cordova [4] discovered that the relationship between planned (simulated) reach distance and actual functional reach was weak. That is, their intentions did not match their actual capabilities and their movement estimation did not align with their actual action execution. Also, others have reported that the elderly experience significant difficulties with the ability to mentally plan and simulate simple and complex, sequential whole-body movements such as walking [5,6]. Taken together, these reports suggest that weak motor planning can prevent a person from determining actions their body needs to perform a motor task correctly and safely.

Other researchers have reported that overestimation of action capabilities in the context of reaching was a common observation among older adults $[7,8]$. Both studies also noted how overestimation of actions could be a major fall risk. As previously stated, a recent review study that looked at the circumstances of falls in elderly people, determined that incorrect transfer or shift of bodyweight was the most frequent cause of falling ( $41 \%$ of all falls) 
[3]. Incorrect transfer or shift of body weight is defined as "selfinduced shifting of bodyweight, causing the center of gravity to move outside the base of support" with the "imbalance [as an] internal rather than eternal [like a slip, trip, or stumble] perturbation." A specific example provided from the study is leaning too far from one's base of support.

Motor imagery is a form of mental representation and the ability to mentally visualize intended actions. More specifically, motor imagery involves visualizing (mentally representing) what a movement feels like, rather than visualizing what a movement looks like. Most of motor programming theories support the view that motor imagery is one of the most important components of effectively planning actions. Motor imagery, also known as kinesthetic imagery, is a rehearsal of movements from an internal or first-person perspective without any actual motion taking place. The key is for one to cognitively represent what the motion feels like, not just what the movement looks like from a first-person perspective. Ultimately, motor imagery is comparative to mental representation and motor planning. Additionally, studies have shown that there is a high association between real and simulated movements [9-12].

Motor imagery practice has been supported as effective in improving motor planning and control [2]. Additionally, evidence has been reported to support the theory that mental practice reinforces abstract mental representation that does not involve effectors [13]. In other words, mental practice, like motor imagery training, reinforces 'central' features of the representation as well as representation of body part processes, such as the hands and fingers. Finally, in a study of the effectiveness of motor imagery practice, practice had a positive effect on actual movement execution [14].

In review, research findings tell us that some older adults lack efficient action planning and the most common reason for falls in the elderly is incorrect transfer or shift of bodyweight, like leaning too far from one's base of support. From these reports, one can reasonably speculate that reaching, especially inefficient reach planning such as over- or underestimation, may cause one to lean too far from their base of support, therefore increasing risk of falling. In other words, many falls in the elderly can be linked to reaching. The intent of the present study was to determine if reachspecific motor imagery training could improve reach planning and potentially reduce fall risk. Since reach estimation was tested in three conditions [seated, standing-on-2-feet, and standing-on-1foot], previous research suggested that participants would perform better while seated. Lastly, it was hypothesized that the younger group (ages 65-73) would show more progress after the training than the adults aged 74-81years.

\section{Materials and Methods}

\section{Participants}

A total of 23 adults between the ages of 65 and 81 years old were recruited from a senior living community in South Texas for this study. Participants did not have any neuromuscular condition that would significantly affect their ability to walk and reach without an assistive device nor had any impairment to visual or auditory acuity. There was one control group (Group 1) made up of 9 participants who did not receive the intervention. The two training groups consisted of Group 2 (65- to 73 years; $n=8$ ) and Group 3(74- to 81 years; $n=6)$. Both intervention groups went through the same process protocol. This study was approved by the University Institutional Review Board.

\section{Assessment of reach ability}

Tests of maximum reach and reach-estimation were administered to each participant prior to the start of and at the completion of the motor imagery training program as a pre- and post-test. The participant completed the tests in the following positions: seated, standing-on-2-feet, standing-on-1-foot. Each condition was first assessed for actual maximum reach, which was used as the comparison for imagined reaches. Actual and imagined reach responses were determined via use of a specialized shortthrow projection system, Sanyo Model PLC-XL5, programmed with Visual Basic for data collection. Visual images were systematically projected onto a table surface at midline $\left(90^{\circ}\right)$. Visual images were projected onto a dark colored tabletop and reach targets consisted of white $2 \mathrm{~cm}$ diameter circles. A fixation point was projected onto a rectangular box (with a 45-degree angle surface) placed at midline approximately $45 \mathrm{~cm}$ from the most distal target. Participants fixated on the point between trials to remove any bias or cue for the response trials.

With the Seated condition, participants sat in a chair aligned with the midline of the table and projected image midline. For the standing (2-and 1- foot) conditions, table height was adjusted to be mid-chest high. With the standing-on-2-feet condition, participants began by positioning both feet comfortably. With the standingon-1-foot condition, participants used their dominant foot to complete the trials. Participants began by standing on both feet with the dominant foot aligned with the midline of the table. When instructed, the non-dominant foot was raised to a comfortable level - approximately 3 inches off the floor. More detail of this general procedure is reported in [15]. The experimental setup was established and conducted in an isolated room at the senior living community resident facility.

Maximum reach was determined by having the participant slide a penny under the middle finger of his or her dominant limb to full extension. This position then had to be maintained for $3 \mathrm{~s}$ without losing balance. The participant was allowed three attempts with the farthest attempt being recorded as their maximum reach. Based on maximum reach, seven imagery targets (2cm diameter) were randomly programmed with one target location representing actual reach, complemented with three target sites farther than the participant's maximum reach (extra personal) and three sites closer than the participant's maximum reach (peri personal). In essence, actual reach was 'scaled' to individual arm lengths, therefore allowing acceptable comparison. Participants were asked 
to focus while using motor imagery to 'feel' themselves (firstperson perspective) executing the movement with their dominant limb. This process encouraged participants to be more sensitive to the biomechanical constraints of the (motor imagery) task.

At the start of each condition, participants placed their hands to their side (standing) or in their lap (seated). Participants were asked to make reach estimation judgments using motor imagery relative to whether the target was within reach ("yes" or "no"). Each participant was trained and provided practice in use of motor imagery. For response trials, data collection began with a 5 s "Ready!" signal-immediately followed by a fixation point lasting 3 s. A target image appeared immediately thereafter and lasted $3 \mathrm{~s}$. Once the target image disappeared an immediate (after imaging) verbal response of "yes" or "no" was required. Target presentation was given in random order with 5 trials at each of the seven targets, for a total of 35 trials.

This entire process was repeated for each of the conditions for each participant in the study. In order to prevent improvement based on practice, the order of the conditions for each participant was randomized.

Motor Imagery Training Program The motor imagery training program lasted 15-60minutes (in progressive increments), 3 times per a week for 4 weeks and followed previously researched recommendations from Gabbard \& Fox [2]. The suggested strategies for designing a motor imagery training program to improve motor representation action planning included:

A. Clear and effective script of instructions. A specific script of instructions needs to be used for the training detailing thought processes and considerations for the participants.

B. Goal-setting. Goal-setting is good practice when trying to accomplish a specific task and could positively impact performance.

C. First-person internalizing. Focusing on performing the action from within oneself. This includes considering and understanding one's own capabilities and possible consequences of movement.

D. Concentration on the effectors. Focusing on the specific body parts that are performing the action.

E. Focus on visual cues (objective/goal). Concentrate on the result (the objective) of the intended action.

F. Reinforcement on kinesthetically feeling execution of movement. Really focus on 'feeling' oneself, rather than 'seeing' oneself perform the movement. This helps promotes effective mental representation.

G. Combine physiotherapy with mental practice. This involves having the person perform the action, not just mental representation of the action. This allows a person to gain a better understanding of their capabilities and possibly allows a person to experience potential consequences of an action.

H. Progress from simple to more complex. To build a foundation and have continued improvement and variety in situations.
I. Practice 15-60 minutes, 3 times per week, for 4 weeks. This is the timeframe commonly suggested and used for many motor training programs regarding other studies.

Suggestions addressed in the training sessions included "combining physiotherapy [actually performing the actions] with mental practice" and "progressing from simple to more complex." For example, "combining physiotherapy with mental practice" was seen when the facilitator had the participant attempt to reach for the object after saying whether they thought they had to overreach, under-reach, or perform neither to successfully reach the object. This provided the participant with experience to assist them in planning and determining the reach action needed for the next exercise. "Progress[ing] from simple to more complex" was seen by gradually changing levels (i.e. seated, standing, reaching up) and objects (i.e. newspaper, pen, paperclip, etc.).

For each training session, the facilitator used a prescribed script for consistency. That script included but was not limited to, strategies like "clear and effective script instructions," "goalsetting," and "reinforcement on kinesthetically 'feeling' execution of movement."

\section{Procedure}

After a general explanation of the study was discussed, all participants were given consent forms to review and sign. After completing the consent form, participants completed a PreScreening Questionnaire that included basic demographic, contact information, addressed questions as to whether the participant met the medical and physical criteria necessary to participate in the study. At the conclusion of these forms, all participants continued to complete the pre-testing of assessment of reach ability in the three conditions (seated, standing-on-2-feet, standing-on-1-foot). The order of conditions was counterbalanced for each participant to account for possible improvement with practice (Figure 1).

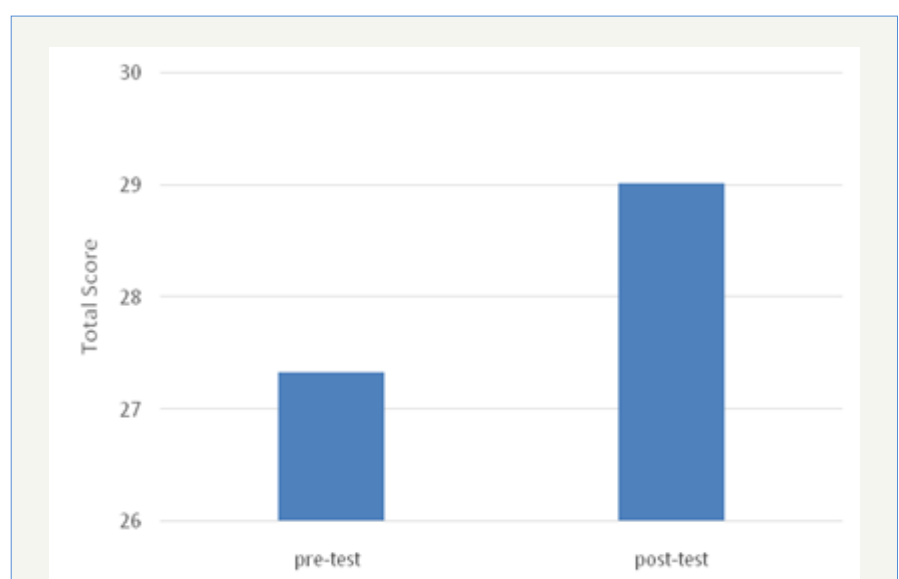

Figure 1: Intervention Group by Time (pre/post) results on Total Score.

The following week, participants in the intervention groups began motor imagery training. The training program, which was previously explained, was conducted 3 times a week (Monday, Wednesday, Friday) for 4 weeks. Participants were encouraged to 
attend the training at the same time each day, but variations were made to correspond with participants' schedules. Sessions were one-on-one beginning with 15 minutes and gradually lengthened in time to no longer than 60 minutes. Each training session began with a relaxation segment, followed by a complete, detailed explanation/ reminder of how the training will be organized, as well as how to use motor imagery throughout the entirety of the session. On the last day of training, all participants completed the post-test assessment of reach-estimation.

Throughout the entire process, participants were instructed to not hesitate to ask if they had any questions or needed clarification. For a detailed copy of the motor imagery training program and sample of the script is available by contacting the corresponding author.

\section{Results and Discussion}

A three-way $2 \times 3 \times 3$ (Time $\times$ Condition x Group) repeated analysis of variance (ANOVA) procedure was used to compare overall accuracy judgment of reach ability. Results for Time revealed a significant main effect (pre-test vs. post-test), $F(1,21)=5.68, \mathrm{p}<.05$. That is, participants had lower total scores in the pre-test condition $(M=27.34)$ compared to the post-test condition ( $M=29.02) ; p<.05$. For Condition (standing, sitting, and one foot), there was no main effect, $F(2,29)=0.09, p=0.77$. That is, participants did not have significantly different scores between conditions. Table 1 shows group mean reach estimation scores for time and condition (Table 1).

Table 1: Mean reach estimation scores for Time and Condition with intervention groups combined.

\begin{tabular}{|c|c|c|c|}
\hline Group & Seated & Standing-on-2-feet & Standing-on-1-foot \\
\hline $\begin{array}{c}\text { Pre- } \\
\text { Intervention }\end{array}$ & 25.21 & 29.07 & 25.5 \\
\hline $\begin{array}{c}\text { Post- } \\
\text { Intervention }\end{array}$ & 28.53 & 29.46 & 28.42 \\
\hline Pre-Control & 27.91 & 27.55 & 29.6 \\
\hline Post-Control & 29 & 29.36 & 29.11 \\
\hline
\end{tabular}

ANOVA results for Group (age), revealed no main effect, $\mathrm{F}(2,42)=1.57, \mathrm{p}=.21$. In other words, there was no significant difference in total scores between the control group $(M=28.76)$, Group $2(\mathrm{M}=27.41)$ and Group $3(\mathrm{M}=28.763)$. There were no significant interactions. Regarding any possible training effect, results indicated no significant difference for the Group 1 (control); pre-test $(M=28.353)$ and post-test $(M=29.15)$. However, there was a significant difference (increase) with both intervention groups combined; pre-test $(M=27.33)$ and post-test $(M=29.02)$, $\mathrm{F}(1,21)=4.89, \mathrm{p}<.05$.

The purpose of this study was to examine the effects of a motor imagery training program on reach estimation in older persons. The main hypothesis predicted that participants would improve in estimation accuracy and speculatively, reduce fall risk in reachspecific contexts. Our findings in part, support that hypothesis, showing that a 4-week motor imagery training program did influence participants' estimation of reach ability (Figure 2).

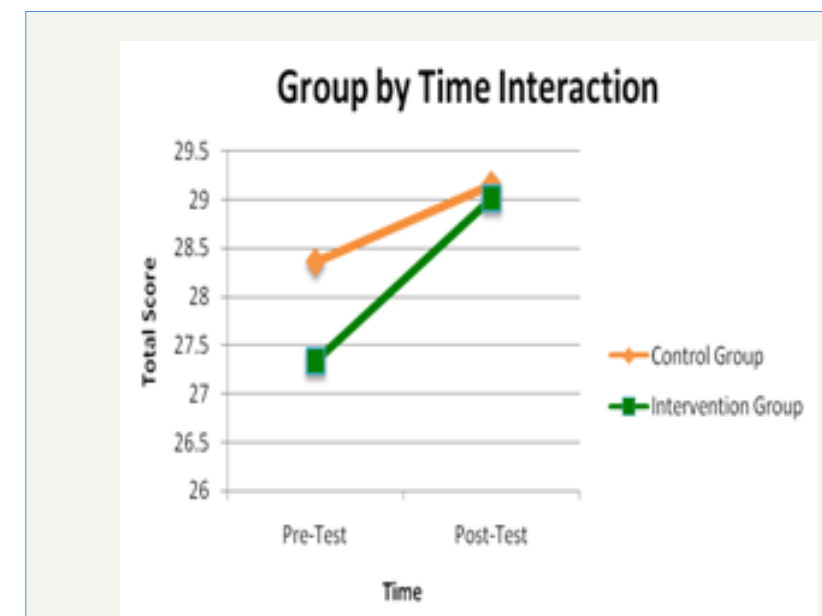

Figure 2: Group by time interaction results.

Regarding reach estimations by condition, interestingly, compared to previous reports [15], there was no significant difference between the three reach positions (sitting, standing on two feet, or standing in one foot). Even though participants were more likely to lose their balance or fall while standing on one foot compared to standing on two feet or sitting down, this instability did not affect their reaching estimations. We can only assume that participants, keeping in mind that they were elderly, chose to be more conservative in their reach ability and underestimated when they felt less stable.

With respect to Age, we did expect a greater improvement over time for the younger Group 2; however, no significant difference between the three groups. These results might have been since the number of participants in each group was too small for a difference to be detected. The sample size was a limitation of the study. Increasing the overall number of participants might decrease the effect that individual differences have in each group, and this might result in more differences between groups due to the training. Therefore, future work is warranted to examine our motor imagery training.

There were many things in this study that could be modified and improved for future research. The study's main limitation, as noted earlier, was the number of participants. Another insight we obtained from our results is that perhaps the training should be longer than just four weeks. With longer training, participants might be able to improve their motor imagery strategies at a more in-depth level. As it was mentioned at the beginning of this research, the elderly population is a growing group in society. Their well-being is a major concern for everyone. Because of this, there is a great need to develop strategies and find solutions to limit the number of falls and injuries that are suffered by these individuals every day. This study, along with previous research, shows that motor imagery training could be a potential solution to this problem and it might lead to a reduction in accidents and fatalities due to falling. If future research continues to find mental imagery 
training successful, this training could be implemented in nursing homes, rehabilitation centers, and senior living facilities. Resulting in lower medical costs and improvement in the everyday living of our senior citizens.

\section{References}

1. Centers for Disease Control and Prevention (2013) Falls among older adults: An overview.

2. Gabbard C, Fox A (2013) Using motor imagery therapy to improve movement efficiency and reduce fall injury risk. Journal of Novel Physiotherapies 3(6): 186.

3. Robinovitch SN, Feldman F, Yang Y, Schonnop R, Leung PM, et al. (2013) Video capture of the circumstances of falls in elderly people residing in long-term care: an observational study. Lancet 381(9860): 47-54.

4. Gabbard C, Cordova A (2013) Association between imagined and actual functional reach: A comparison of young and older adults. Arch of Gerontol Geriatr 56(3): 487-491.

5. Saimpont A, Mourey F, Manckoundia P, Pfitzenmeyer Pierre, Pozzo T (2010) Aging affects the mental simulation/planning of the "rising from the floor" sequence. Arch Gerontol Geriatr 51(3): e41-e45.

6. Saimpont A, Malouin F, Tousignant B, Jackson PL (2013) Motor imagery and aging. J Mot Behav 45(1): 21-28.

7. Gabbard C, Cacola P, Cordova A (2011) Is there an advanced aging effect on the ability to mentally represent action? Arch Gerontol Geriatr 53(2): 206-209.
8. Noël M, Bernard A, Luyat M (2011) The overestimation of performance: A specific bias of aging? Geriatr Psychol Neuropsychiatr Vieil 9(3): 287294.

9. Burianová H, Marstaller L, Sowman P, Tesan G, Johnson B, et al. (2013) Multimodal functional imaging of motor imagery using a novel paradigm. NeuroImage 71: 50-58.

10. Kuntz BR, Creem-Regehr SH, Thompson WB (2009) Evidence for motor simulation in imagined locomotion. J Exp Psychol: Hum Percept Perform 35(5): 1458-1471.

11. Lorey B, Bischoff M, Pilgramm S, Stark R, Munzert J, et al. (2010) The embodied nature of motor imagery: The influence of posture and perspective. Exp Brain Res 194(2): 233-243.

12. Lorey B, Nauman T, Pilgramm S, Peterman C, Bischoff M, et al. (2013) How equivalent are the action execution, imagery, and observation of intransitive movements? Revisiting the concept of somatotopy during action simulation. Brain Cogn 81(1): 139-150.

13. Wohldmann EL, Healy AF, Bourne LE (2008) A mental practice superiority effect: Less retroactive interference and more transfer than physical practice. J Exp Psychol Learn Mem Cogn 34(4): 823-833.

14. Guttman A, Burstin A, Brown R, Bril S Kickstein R (2012) Motor imagery impact for improving sit and stand and reaching to grasp in individuals with poststroke hemiparesis. Top Stroke Rehabil 19(4): 306-319.

15. Cordova A, Gabbard C (2014) Do older adults perceive postural constraints for reach estimation? Exp Aging Res 40(5): 578-588. 